\title{
An Investigation of the Visual-Mental Capability of Pre- and In-Service Mathematics Teachers: A Tale of Two Cones and One Cube
}

\author{
BARKAI,Ruthi \\ Kibbutzim College of EducationTechnology and the Arts, \\ 36 Remez Street, Tel Aviv, Israel; Email: ruthi11@netvision.net.il \\ PATKIN, Dorit ${ }^{*}$ \\ Kibbutzim College of EducationTechnology and the Arts, \\ 13 Dakar Street, Beit Yanai, Israel; Email: patkin@netvision.net.il
}

(Received December3, 2013;Revised December 8, 2013; Accepted March21, 2014)

\begin{abstract}
This study investigated the visual-mental capabilityof pre-service and in-service mathematics teachers as well as academicians making a career change to mathematics teachers with regard to manipulations of two geometric shapes (from 2- to 3-dimensional). Moreover, it investigated whether there are differences between the visual-mental capabilityof these participant groups. Findings illustrate that most of the participants demonstrate an adequate visual capability relating to the task dealing with a cube. Conversely, very low percentage of participants manifested a visual-mental capability in a task requiring the identification of a solid resulting from rotation of a square page, whose diagonal serves as the rotation axis. The study indicates that learners' high visual view should be developed in order to enhancetheir visual-mental capability.
\end{abstract}

Keywords: visual geometric thinking, solid geometry, spatial thinking, pre-service and in-service mathematics teacher education,academicians making a career change to mathematics teachers.

MESC Classification: B54, B55, B59, G42, G43, G44, G45, G49

MSC2010Classification:97B50, 97G40

\section{INTRODUCTION AND THEORETICAL BACKGROUND}

Mathematics educators concur about the importance of developing spatial orientation and visual-mental capability for the enhancement of mathematical thinking in general and

\footnotetext{
* Corresponding author
} 
visual thinking in particular(Patkin\& Dayan, 2014; Halpern, 2005; Sorby, Wysocki\&Baartmans, 2002).

Solid geometry is one of the topics of geometry studied from kindergarten, throughout the years in elementary school, continues at high school and up to mathematics teacher education in college. Quite a few studies explored children's thinking related to spatial orientation in general and perception of concepts associated with spatial geometric shapes in particular (Shaw 1990; Yackel\& Wheatley, 1991; Hannibal, 1999). Spatial comprehension comprises two components, visual-mental capability and spatial orientation. Spatial orientation is the position of an object in space with regards to a frame of reference that is chosen. Visual-mental capability is the ability to imagine 2-dimensional shapes and solids as well as their movement or change in theirattributes(Del Grande, 1990). Developing visual-mental capability is based on learners' experiences and activities with 3D shapes, using illustration aids and actual experience of building 3D shapes (Patkin \& Sarfaty, 2012). The National Council of Teachers of Mathematics (NCTM, 2000) defined several standards, principles and tools for teaching mathematics at different ages. The document explicitly states that the development of visualisation is one of the tools for solving mathematical problems:"Students should gain experience in using a variety of visual and coordinate representations to analyse problems and study mathematics...beginning in the early years of schooling, students should develop visualisation skills through hands-on experiences with a variety of geometric objects...students need to learn physically and mentally to change the position, orientation and size of objects.... One aspect of spatial visualisation involves moving between 2- and 3-dimensional shapes and their representations...."(NCTM, 2000, pp, 42-43).

Walker, Winner, Hetland, Simmons \& Goldsmith (2011) argue that, although the importance of visual-mental capability is recognised in the learning of mathematics, solid geometry is usually studied with a strong emphasis on the formal aspect and symbolic representation rather than on visual-mental capability(Walker et al., 2011). Teaching which integrates formal aspect and visual aspect facilitates complete conceptualisation of geometric ideas and the ability to cope with geometric assignments (Walker et al., 2011). This attitude is supported by the work of the mathematician William Thurston, winner of the Fields Medal in 1982. He became famous after illustrating the power of visual representations in the conceptualisation of abstract mathematical ideas. He claimed that using visual tools for representing abstract mathematical ideas contributes more than formal proofs. Consequently, he developed ways for teaching geometry by means of visual arguments (Horgan, 1993). According to Presmeg (1986) and Bishop (1989), the ability to create mental pictures may improve the understanding of mathematics concepts and problem solving. In her study, Cohen (2007) discussed the analysis of geometrical thought processes of pre-service teachers and in-service teachers while performing tasks in the 3- 
D space. Her main goal was to identify supporting and hindering factors which affect the cognitive processes, and to examine the effect of combining visual imaging and analytical difficulties and misconceptions. Cohen's findings supported the premise that strengthening the use of analytical-visual integration enables improvement of the ability to imagine pictures. Strengthening the analytical-visual connection facilitates a more comprehensive understanding of concepts and space relations. Walker et al. (2011) investigated whether students of art have high visual-mental capability, because art is an area of knowledge requiring high visual-mental capability. Their study consisted of two student groups: 18 art students and 18 psychology students. The participants were asked to respond to 27 items designed to check visual-mental capability. Answering these items did not depend on the students' formal knowledge of geometry, such as definitions, theorems and proofs, but required 2- and 3-dimensional visual-mental capability. Findings of the study conducted by Walker et al. (2011) showed that the art students demonstrated better capabilities in solving the geometric assignments and that there was a considerable difference in their visual-mental capability versus those of the psychology students. All these findings corroborate the researchers' claim that developing visual-mental capability in art improves geometric thinking.

Hence, it is very important that teachers and pre-service teachers are aware that using a variety of assignments engaging in visual-mental capability as well as shifting from 2to 3-dimension and vice versa in varied disciplines which learners study, can facilitate the development of students' spatial orientation, bringing them to an advanced level of geometric thinking.

The study presented in this paper focuses on mathematics education and, therefore, deals with the visual-mental capability of pre and in-service teachers. Two assignments in solid geometry were chosen for testing the visual-mental capability of the research participants (three groups of participants: pre-service and in-service teachers as well as careerchanging academicians) to cope with those assignments. Moreover, the study explored whether there are difference in the way of thinking of the three groups of participants.

\section{Research question}

1. What is the visual-mental capability of the research participants (pre-service, 1st year, B.Ed. and in-service teachers as well as career-changing academicians) regarding manipulations of geometric shapes (from 2-dimensional to 3-dimensional)?

2. Are there any differences in the visual-mental capability of in-service mathematics teachers, pre-service mathematics teachers and academicians making a career change at different points during their education (pre-service, 1st year, B.Ed. and in-service teachers as well as career-changing academicians)? 


\section{METHODOLOGY}

\section{Research population}

The research population consisted of 87 participants, learning in an academic college of education. The participants were divided into three different sub-groups. The first subgroup comprised 46 pre-service teachers in their first year of studying to become elementary school mathematics teachers. These participants have never taken any academic geometry course on the topic of solids (3D). The second sub-group consisted of 18 participants with a B.A. or M.A. in another area of knowledge, making a career change to mathematics teachers at elementary and junior high school. These academicians are in their first year (out of two years) of studies for obtaining a teaching certificate. The third subgroup included 23 in-service mathematics teachers studying towards an M.Ed. in mathematics education for elementary school. These teachers have B.Ed. in mathematics and teach geometry including the topic of solids (3D) at their school.

\section{Research tools}

The research tool was a questionnaire with two assignments, chosen out of the 27 assignments developed by Walker et al. (2011).

Based on our experience as lecturers and researchers we are aware that, sometimes,while coping with assignments, pupils improve their knowledge. Consequently, in order to comprehend their real knowledge, we decided to present only two assignments, knowing that exposure to a wide variety of assignments might be a kind of exercise or intervention which we wanted to avoid at this stage of the study. Moreover, we did not want to put too much burden on the respondents who might refuse to answer the questions relating to these assignments in a well-argumented and detailed way. As already mentioned, these assignments do not depend on formal knowledge of geometry, e.g. definitions, theorems and proofs but rather focus on geometric thinking.

These assignments require reliance on vision and ability to imagine solids. In order to investigate the in-service and pre-service teachers' ability to solve the assignments by mental imaging, without manipulation of external representations, the participants were asked to respond to the assignment in as detailed a manner as possible, using words and without using a drawing. In case they used a drawing, they were asked to explain why they did it, indicating at what stage of the answering process they decided to use a drawing (prior to the solution, during the solution or as a backup of the solution they suggested).

The two assignments (detailed further on) were chosen so that one assignment dealt 
with an unfamiliar rotated solid (solid consisting of two cones with a common basis) whereas the second dealt with a familiar solid (a cube). That is, we wanted to check whether there was a difference in the respondents' answers and their visual capabilities in these two assignments.

Below are the two assignments.

First assignment: imagine you are holding in your hand a paper square, holding it at two opposite vertices. Now rotate the square so that the diagonal between those vertices constitutes the rotation axis. Which image of a shape is created?

Second assignment: imagine a cube. You are asked to paint all the faces of the cube so that no adjacent faces are of the same colour. What is the minimal number of colours required, in your opinion, to complete the assignment?

The first assignment requires rotating a square page whose diagonal serves as the rotational axis, creating a 3-dimensional solid comprised of two cones with a common basis.

It is important to point out that rotating an isosceles right-angled triangle around the altitude to the basis of the triangle creates an image of a cone (3-dimensional figure).
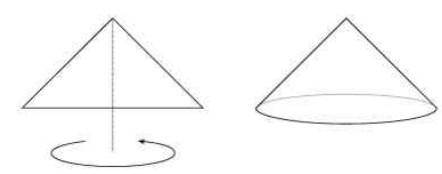

\section{Illustration 1a.Rotation of an isosceles right-angled triangle}

The diagonal of the square divides it into two congruent isosceles triangles. Hence, rotating a square page around its diagonal creates a solid consisting of two cones with a common basis.

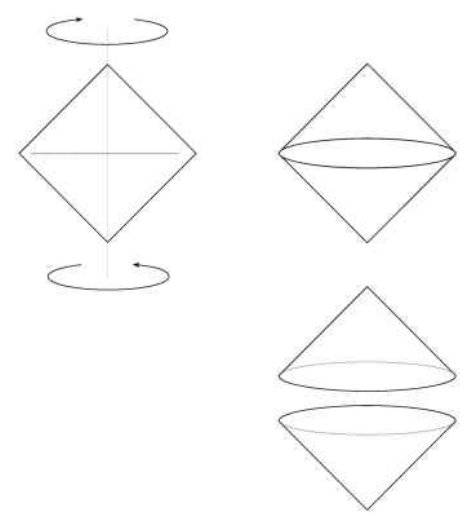

Illustration 1b.Rotating a square page around its diagonal 
In the second assignment, respondents are required to imagine a solid (cube) familiar to them, on which mental manipulation should be performed (painting the faces of the cube) and then to explain their answer.

The assignment focused on the minimal number of colours required for painting the faces of the cube so that no two adjacent faces are in the same colour. A cube has six faces and each face is adjacent to four other faces of the cube. Consequently, the minimal number of colours, according to the requirements of the assignment, is three.

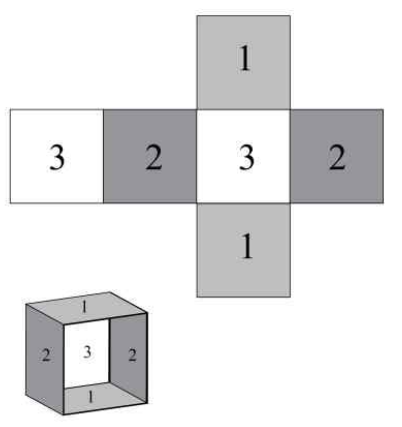

Illustration 2. The cube

\section{Research procedure}

The questionnaire was administered to each group as part of the end-of-course exam in the geometry courses they had studied, according to their year of education and different pathways ( $1^{\text {st }}$ year, B.Ed., M.Ed., career change). The courses were taught by the researchers of the present study. A full answer to each of these two assignments awarded the respondents 5 points (a total of 10 points).

The respondents could decide for themselves in which order the assignments would be solved.

\section{Ethical considerations}

Permission to conduct the study was granted by the college. The participants gave their consent to take part in the study. The aims and objectives of the study were discussed with them. The name of the college and those of the learners were not divulged.

\section{FINDINGS}

Table 1 presents the distribution (\%) of correct and incorrect answers given by the participants to the first assignment: identifying the solid resulting from the rotation of a 
square page, whose diagonal serves as the rotation axis (the obtained solid is built of two cones with a common basis). Moreover, the table indicates the participants who did not respond to this assignment. The table refers to each of the three sub-groups participating in the study: pre-service elementary school mathematics teachers in their first year of studies; in-service elementary school teachers studying towards an M.Ed. in mathematics education; and academicians making a career change to mathematics teachers at elementary and junior high school.

Table 1.Distribution (\%) of answers in the cones assignment

\begin{tabular}{|c|c|c|c|c|}
\hline Sub-Group & $\mathbf{N}$ & Correct(\%) & Incorrect(\%) & Did notrespond \\
\hline 1st year & 46 & 0 & $41(89 \%)$ & $5(11 \%)$ \\
\hline Career change & 18 & $7(39 \%)$ & $6(33 \%)$ & $5(28 \%)$ \\
\hline M.Ed. & 23 & $4(17 \%)$ & $14(61 \%)$ & $5(22 \%)$ \\
\hline Total & $\mathbf{8 7}$ & $\mathbf{1 1}(\mathbf{1 3 \%})$ & $\mathbf{6 1}(\mathbf{7 0} \%)$ & $\mathbf{1 5}(\mathbf{1 7 \%})$ \\
\hline
\end{tabular}

Table 1 illustrates that only $13 \%$ of the participants in this study gave a correct answer and identified that the resulting solid was two cones with a common basis. These were the academicians making a career change to mathematics teachers at elementary and junior high schools and in-service teachers studying for M.Ed. in elementary school mathematics education. $89 \%$ of the pre-service teachers in their first year of studying to become elementary school mathematics teachers did not respond correctly to this assignment. The other $11 \%$ failed to respond to this assignment. Among those learning towards an M.Ed. and the career-changing academicians, the percentage of non-respondents was twice higher and even more than that (22\% and $28 \%$ respectively).

As for the participants who answered this assignment correctly, the findings show that only five (out of the 11 who responded correctly) offered arguments. All the arguments presented were correct and related to essential attributes of the created solid. The participants pointed out that one diagonal of the square is the diameter of the obtained cones whereas the second diagonal constitutes the altitudes of these cones. The sides of the square are the creating line segments of the cones. Below are the explanations given by the participants.

\footnotetext{
"The diameter is the diagonal of the square. The creating line segment is the side of the square" [In-service teacher R]; "The radius of their bases is half the diagonal of the square and their altitude is half of the diagonal of the square and you connect them at their bases" [In-service teacher M]; "The ends of the square (the vertices which we are not holding) create a circular basis (collection of dots equally positioned from the centre of the square which becomes the centre of the circle" [In-service teacher A]; "The side of the square will become the creating line segment of the cone on each side" [Career change $\mathrm{C}$;; "The vertex of the cones will be the opposite vertices of the cube which are held in both hands. The rotation around the diagonal axis of the square turns the di-
} 
agonal of the cube in the connection of the two cone altitudes" [Career change F].

It is worth noting that some of the participants, including those who did not give any mathematical argument, described their decision-making process regarding the (correct) answer.

The participants indicated that for the purpose of making a decision or checking their answer, they used a tangible item available to them such as a notebook, a notebook page or a handkerchief. They did not rely only on the mental picture of the solids.

\begin{abstract}
"I usedthe examination page in order to see what we get.It took me time to understand the problem. Only after rotating the page several time I saw these were two cones connected at the basis" [In-service teacher H]; "It is interesting to point out! Rotation of a square is done in the head, but as backup I rotated the exam notebook" [Career change O]; "I used a drawing and, mainly, a handkerchief in order to comprehend the meaning of rotating around one axis while the other axis (the second diagonal) is turning around" [In-service-teacher S].
\end{abstract}

Table 2 presents the variety of incorrect answers given by the participants to the first assignment. The table relates to the distribution of the incorrect answers about 3dimensional solids and to the distribution of incorrect answers about 2-dimensional shapes.

Table 2.Distribution of incorrect answers in the cones assignment

\begin{tabular}{|c|c|c|c|c|c|}
\hline \multirow[t]{2}{*}{ Figure } & The answer & $\begin{array}{c}1 \text { st } \\
\text { year }\end{array}$ & $\begin{array}{l}\text { Career } \\
\text { change }\end{array}$ & M.Ed. & Total \\
\hline & & $N=41$ & $N=6$ & $N=14$ & $N=61$ \\
\hline \multirow{8}{*}{$3-\mathrm{D}$} & Cone & 3 & --- & 1 & 4 \\
\hline & Cylinder & 12 & --- & 1 & 13 \\
\hline & Ball & --- & 3 & 3 & 6 \\
\hline & Pyramid & --- & --- & 2 & 2 \\
\hline & Cube & 2 & 1 & 1 & 4 \\
\hline & Square prism - box & 2 & --- & --- & 2 \\
\hline & $\begin{array}{l}2 \text { square pyramids with a } \\
\text { common basis - octagon }\end{array}$ & 4 & --- & 3 & 7 \\
\hline & A very thin body & 1 & --- & 2 & 3 \\
\hline \multirow{7}{*}{$2-\mathrm{D}$} & Square & 4 & --- & 2 & 6 \\
\hline & Circle & 2 & --- & --- & 2 \\
\hline & Triangle & 3 & 2 & 1 & 6 \\
\hline & Rhombus & 4 & --- & --- & 4 \\
\hline & Kite & 2 & --- & --- & 2 \\
\hline & Parallelogram & 1 & --- & --- & 1 \\
\hline & Other & 1 & --- & --- & 1 \\
\hline
\end{tabular}


Table 2 shows that 39 incorrect answers related to solids and 22 answers related to incorrect shapes. Among the $1^{\text {st }}$ year participants, 13 types of incorrect answers were found. The most prevalent incorrect answer was the cylinder. This answer might have been given based on the knowledge that rotating a rectangular paper page around one of its sides results in a cylinder. This answer did not relate to the fact that the rotation axis in the assignment was not one of the square sides but rather its diagonal.Additional five types of incorrect solids (10 answers) and seven types of incorrect shapes (17 answers) were presented by the participants of this sub-group. Among the academicians making a career change to mathematics teachers, three types of incorrect answers were given.Half of the academicians (three out of six) indicated that the created solid would be a ball. Another type of incorrect solids (one answer) and one type of incorrect shape (two answers) were also presented by the participants of this sub-group. The in-service teachers studying for an M.Ed. presented a variety of eight incorrect types of answers: eleven participants of this sub-group related in their incorrect answer to different solids and three others related to two types of 2-dimensional shapes.

Table 3 indicates the distribution (\%) of correct and incorrect answers which the participants gave to the second assignment. In that assignment they were required to find the minimal number of colours necessary for painting all the cube's faces so that there would be no adjacent faces of the same colour (the minimal number of colours is three). Furthermore, the table presents the distribution of participants who failed to respond to that assignment. The table relates to each of the groups participating in the study.

Table 3. Distribution (\%) of answers in the cube assignment

\begin{tabular}{|l|c|c|c|c|}
\hline \multicolumn{1}{|c|}{ Sub-Group } & $\mathbf{N}$ & Correct(\%) & Incorrect(\%) & Did not respond \\
\hline 1st year & 46 & $40(85 \%)$ & $5(11 \%)$ & $1(4 \%)$ \\
\hline Career change & 18 & $15(83 \%)$ & $1(6 \%)$ & $2(11 \%)$ \\
\hline M.Ed. & 23 & $20(87 \%)$ & $1(4 \%)$ & $2(9 \%)$ \\
\hline Total & $\mathbf{8 7}$ & $\mathbf{7 5 ( 8 5 \% )}$ & $\mathbf{7 ( 8 \% )}$ & $\mathbf{5 ( 7 \% )}$ \\
\hline
\end{tabular}

Unlike the findings relating to the first assignment, Table 3 illustrates that almost all the participants $(85 \%)$ responded to this assignment. The percentage of participants in each of the three groups in the present study who gave a correct answer was similar (83\%-87\%). We believe that the differences in the participants' ability to give a correct answer to the cube assignment, as compared to their ability to answer the cones assignment, stem from the fact that the cube assignment presented a solid which is familiar to them and they could easily imagine it.

All the participants, with the exception of five who responded correctly to the second assignment (the cube assignment), presented an argument for their (correct) answer. The 
arguments which the participants presented were similar. There was no difference between the arguments presented by the in-service teachers studying for an M.Ed. and the pre-service teachers in their first year of studies or the academicians making a career change to elementary and junior high school mathematics teachers.

Relating to the fact that every two parallel faces could be painted in the same colour and a cube has three pairs of parallel faces.

"A cube has 6 faces, each one is parallel (and congruent) to another face. Hence, two parallel faces will be painted in the same colour. A cube has three such pairs and, therefore, three colours are needed" [Pre-service P]; "The minimal number of colours for performing the assignment is three - one colour for each opposite (and parallel) pair of faces" [In-service teacher G].

Most of the arguments highlighted that every side of the cube is adjacent to four faces and not only to one face of the cube, i.e. two such faces can be painted in the same colour. Consequently, the participants concluded that every two (parallel) faces can be painted in the same colour and that a cube has three such pairs.

"A cube has six faces and each face is adjacent to four faces and not only to the faces opposite it. Therefore, if we divide six by two the answer will be three" [In-service teacher N]; "Every face of the cube 'touches' four other faces. However, out of the four faces it touches, two are opposite. That means that only three colours are necessary $(6: 2=3)$ "'[In-service teacher R]; "A cube has six faces and each face has common edge with other four faces, namely there is only one side which is not adjacent to it. So, two parallel faces will be painted in the same colour" [Pre-service teacher J].

Regarding the incorrect answers given to this assignment (a total of seven participants), five of them argued that five colours would be needed.

"One needs five colours because every face has four adjacent faces. One can choose a pair of opposite faces and paint them in the same colour, painting the four additional faces in different colours" [Pre-service K]; "five colours. Four faces adjacent to one another and the two bases are not adjacent. Therefore we need one colour for the bases and four different colours for the envelope" [Career change L].

These arguments do not take into consideration that the face they choose is random and that this argument applies to any chosen face of the cubes. Thus, every pair of parallel faces can be painted in the same colour.

One participant maintained that four colours should be sufficient.

"Every face 'touches' four different faces and, therefore, we need four colours" [Preservice I]. Yet another participant claimed that "we need six colours since the cube has six faces" [In-service A]. 


\section{DISCUSSION AND CONCLUSIONS}

This study focuses on mathematics education and, therefore, engages in the knowledge of pre-service and in-service teachers. This population has been extensively researched in studies of mathematics education with the purpose of exploring the knowledge and capabilities of those engaged in the teaching of mathematics.

The research aims of the present study were to explore the visual-mental capability of pre-service and in-service mathematics teachers as well as of academicians making a career change to mathematics teachers regarding manipulations of geometric shapes.

Furthermore, the study examined whether there are differences in the visual-mental capability of these participant groups.

Only a few studies investigated the visual-mental capability of pre-service and inservice teachers at different stages of their education regarding manipulations of geometric shapes and transition from 2-dimensions to 3-dimensions. Studies focusing on the relation between visual capabilities (e.g. of art students) and formal aspect of geometry illustrate that visual art can serve as a starting point or even as a jumping board for learning geometry and enhancing geometric comprehension. Visual capability can be a useful tool for promoting geometric comprehension, mainly when focusing on dynamic geometric thinking instead of memorisation and application of static rules and geometric relations (Seago, Driscoll \& Jacobs, 2010; Walker et al., 2011).

The study conducted by Walker et al. (2011) investigated students of psychology and art in order to check whether the visual-mental capabilities to answer assignments dealing with manipulation of shifting from 2-dimensions to 3-dimensions are different between art and psychology students. The present study, however, aimed to examine the visualmental capabilities of pre-service and in-service mathematics teachers with regard to these unusual assignments which are not studied directly in the curriculum.

All the participants in this study have high school education, including matriculation in mathematics. They were directly or indirectly exposed to solid geometry during their previous studies (the differences reside in the number of mathematics learning units they took at high school).

Findings of the present study show that the participants demonstrated low visualmental capability in a less familiar assignment which is not so prevalent in their professional world: identifying the solid created by rotating a square around its diagonal (13\% answered correctly and 70\% answered incorrectly). This finding is typical of all the participants of the present study regardless of the sub-group to which they belonged. In the familiar and common assignment, which the participants encounter during their studies or teaching in practice, most participants $(85 \%)$ manifested a high visual-mental 
capability. Similarly to the first assignment, in this assignment too no differences between the sub-groups of the research populations were found.

The findings indicate that in the cube assignment only few participants were wrong $(8 \%$ responded incorrectly and $7 \%$ gave no answer at all). Based on the findings, we maintain that the participants' typical mistake was due to several reasons: they failed to master the cube attributes; they understood the cube attributes, but failed to apply these to the issue of non-adjacent sides of a cube; they oversimplifiedthese attributes, saying that a cube has six faces, therefore six colours are required.

In the cones assignment, the participants presented a variety of incorrect answers ( $70 \%$ and $17 \%$ did not respond). Some of the mistakes were characterised as a misconception in the transition from a 2- to 3-dimensional shape. Many of these participants related to a cylinder or cone on the one hand or to solids whose basis is not round, such as box or cube on the other. Another typical misconception was that the rotation had no effect on the shape dimensions, namely the participants perceived that the created shape was 2dimensional following the rotation.

In light of the difficulties encountered by the pre-service teachers, in-service teachers and the career-changing academicians in the two solid geometry assignments, we believe that using operational ideas such as the assignments included in the present study can develop learners' spatial orientation as well as help those engaged in the teaching of geometry to bring learners to an advanced level of geometric thinking.

Findings of the present study illustrate the importance and contribution of visualmental capability to geometric thinking, even in studies of another area of knowledge, art for example. Hence, it is recommended integrating in geometry teaching activities which nurture and develop a visual-mental capability at all stages of learning and in different pathways, from kindergarten to academic education. Learners' visual-mental capability might be developed not only within the framework of geometry teaching but in other areas as well and, thus, their spatial orientation and geometric thinking would be enhanced.

These recommendations are corroborated also by studies (Gardner, 2007; Walker et al., 2011) which advocate encouraging art teachers to collaborate with their colleagues in various academic fields, while applying knowledge from one field in another field. This collaboration might provide comprehensive education in line with the $21^{\text {st }}$ century spirit also for art students and other academicians, preparing them for the future by teaching them skills which would enable them to make a synthesis of various areas of knowledge. 


\section{ACKNOWLEDGEMENTS}

\section{Competing interests}

The authors declare that they have no financial or personal relationships which may have inappropriately influenced them in writing this article.

\section{REFERENCES}

Bishop, A. J. (1989). Review of research on visualization in Mathematics Education, Focus Learn. Probl.Math.11(1), 7-16. ME19921.00230

Cohen, N. (2007). Analytical-Visual Integration in 3-D Tasks. Thesis submitted for the degree of "Doctor of Philosophy", Beer-Sheba, Israel; Ben-Gurion University of the Negev.

Del Grande, J. (1990). Spatial Sense.Arith.Teacher.37(6), 14-20. ME1990i.37157

Gardner, H. (2007). Five Minds for the Futuere. Cambridge, MA: Harvard Business School Press.

Halpern, F. D. (2005). Sex, Brains \& Hands - Gender Differences in Cognitive Abilities. Skeptic, 2(3), 96-103.

Hannibal, M. A. (1999). Young Children's Developing Understanding of Geometric Shapes. Teach. Child.Math.5(6), 353-357. ME2000b.01200

Horgan, J. (1993). The death of proofs.Scientific American, 92-103. Retrieved From:April 2012http://www.math.uh.edu/ tomforde/Articles/DeathOfProof.pdf.

National Council of Teachers of Mathematics (NCTM).Principles and standards for school mathematics. Reston, VA:NCTM. ME1999f.03937 for discussion draft (1998)

Patkin, D. \& Dayan, E. (2013).The intelligence of observation improving high school students' spatial ability by means of intervention unit.Int. J. Math. Educ. Sci. Technol. 44(2), 179-195. ME2013c.00191 http://dx.doi.org/10.1080/0020739X.2012.703335

Patkin, D. \& Sarfaty, Y. (2012).The effect of solid geometry activities of pre-service elementary school mathematics teachers on concepts understanding and mastery of geometric thinking levels.J. Korean Soc. Math. Educ., Ser. D, Res. Math. Educ.16 (1), 31-50.

Presmeg, N. (1986). Visualization in high school mathematics. Learn. Math. 6(3), 42-46. ME1987f.03327

Seago, N.; Driscoll, M. \& Jacobs, J. (2010). Transforming Middle School Geometry: Designing Professional Development Materials that Support the Teaching and Learning of Similarity.Middle Grades Research Journal5(4), 199-211

Shaw, J. M. (1990). By Way of Introduction. Arith.Teacher.37(6), 4-5.

Sorby, S. A.;Wysocki, A. F. \&Baartmans, B. G. (2002). Introduction to 3D Spatial Visualisa- 
tion.New York: Cengage Learning.

Walker, C. M.; Winner, E.;Hetland, L.; Simmons, S. \& Goldsmith, L. (2011).Visual Thinking: Art Students Have an Advantage in Geometric Reasoning. Creative Education2(1), 22-26.

Yackel, E. \& Wheatley, G. H. (1990). Promoting Visual Imagery in Young Pupils. Arith.Teacher.37

(6), 52-58. ME1990i.371164 